\title{
USO DE HÁBITAT Y RELACIONES ECOMORFOLÓGICAS DE UN ENSAMBLE DE ANOLIS (LACERTILIA: DACTYLOIDAE) EN LA REGIÓN NATURAL CHOCOANA, COLOMBIA
}

\section{JHON TAILOR RENGIFO M., ${ }^{1}$ FERNANdo CASTRO HERRERA² Y FrANCISCO JosÉ PURROY IRAIZOS ${ }^{3}$}

\author{
${ }^{1}$ Universidad Tecnológica del Chocó, Grupo de Herpetología, Facultad de Ciencias Naturales, Chocó-Colombia \\ $<$ jhontailorrengifo@gmail.com> \\ ${ }^{2}$ Universidad del Valle, Laboratorio de Herpetología, Cali, Valle del Cauca-Colombia <fcastro2000@gmai.com \\ ${ }^{3}$ Universidad de León, Departamento de Biodiversidad y Gestión Ambiental, León-España. <fjpuri@unileon.es>
}

Recibido: 10/07/2013; aceptado: 10/02/2015

Rengifo M., J. T., Castro Herrera, F. \& Purroy Iraizos, F. J. 2015. Uso de hábitat y relaciones ecomorfológicas de un ensamble de Anolis (Lacertilia: Dactyloidae) en la región natural Chocoana, Colombia. Acta Zoológica Mexicana (n. s.), 31(2): 159-172.

RESUMEN. Se evaluó el hábitat usado por un ensamble de Anolis en bosque pluvial tropical del Chocó-Colombia, usando el método de Encuentros Visuales (Visual Encounter Survey, VES) en tres coberturas vegetales. Se encontró una diferencia estadística en la posición vertical $\left(X^{2}=58.7, \mathrm{gl}=3, P<0.0001\right)$, sustratos $\left(X^{2}=272.1, \mathrm{gl}=4, P<0.0001\right)$ y en el diámetro de percha $\left(X^{2}=147.5\right.$, gl $\left.=6, P<0.0001\right)$ además, se encontró que $A$. maculiventris y $A$. anchicayae son las especies de mayor IVI con 0.69 y 0.51 (33 y 25\%), respectivamente. Se midió el valor de importancia de las especies en las diferentes coberturas vegetales. En este estudio sobresalieron las especies de ecomorfo tronco-rama (A. malkini, A. chloris, A. latifrons, A. lyra y A. notopholis); todas las especies ocurren en los troncos de los árboles y arbustos, y especies como A. maculiventris y A. granuliceps se pueden encontrar ocupando microhábitat en el suelo generalmente en los troncos caídos y la hojarasca; las variables climáticas medidas no mostraron una correlación fuerte con las abundancias registradas.

Palabras clave: Hábitat, Anolis, Chocó, Bosque, Ensamble.

\section{INTRODUCCIÓN}

La diversidad y riqueza del género Anolis en ecosistemas tropicales y la forma que utilizan o se distribuyen en la estructura de la vegetación son objeto de interés para estudios de manejo tendientes a la conservación de los hábitat, debido al evidente deterioro ocasionado por factores antrópicos como la fragmentación, contaminación y cambio de uso del suelo para cultivos y potreros. Uno de los enfoques que se está aplicando es que se le considere grupo parámetros para tener una aproximación sobre los efectos que producen los cambios en el uso de suelos, y sus impactos en la diversidad de grupos particulares, sin importar el grado de conservación de los hábitats (Halffter 1994). Muy pocos trabajos están dedicados al conocimiento de comunidades, haciendo énfasis en el uso del hábitat, se resaltan los trabajos de Moermond (1979), Estrada \& Silva (1984), Pounds (1988), Castro (1988), Losos \& Sinervo (1989), Elstrott \& Irschick (2004), Molina \& Gutiérrez (2007), Calsbeek et al. (2007), Velasco
Rengifo M., J. T., Castro Herrera, F. \& Purroy Iraizos, F. J. 2015. Habitat use and ecomorphology relation of an assemblage of Anolis (Lacertilia: Dactyloidae) in the Chocoan natural region from Colombia. Acta Zoológica Mexicana (n. s.), 31(2): 159-172.

ABSTRACT. The habitat used by Anolis in tropical rainforest was evaluated using the Visual Encounters Survey method (VES) in three vegetation cover, there was a statistical difference in vertical position $\left(X^{2}=58.7, \mathrm{df}=3, P<0.0001\right)$, substrates $\left(X^{2}=272.1, \mathrm{df}=4, P<\right.$ $0.0001)$ and perch diameter $\left(X^{2}=147.5\right.$, $\left.\mathrm{df}=6, P<0.0001\right)$. It was found that $A$. maculiventris and $A$. anchicayae are the species with largest IVI with 0.69 and 0.51 (33 and 25\%) respectively, measuring the importance value of species in different vegetation cover. This study highlights ecomorph species trunk-branch (A. malkini, A. chloris, A. latifrons, A. lyra and A. notopholis), all species occur on the trunks of trees and shrubs, and species such as A. maculiventris and $A$. granuliceps microhabitat can be found on the ground usually occupying fallen logs and litter; climatic variables measured did not show a strong correlation with recorded abundances.

Key Words: Habitat, Anolis, Chocó, Forest, Assemblage.

\& Herrel (2007), Hite et al. (2008), Leal et al. (2008) y Traverzo-Pérez (2008). En el presente trabajo se estudia la estructura del hábitat y la forma de uso del microhabitat de especies del género Anolis en zonas de bosque pluvial tropical del departamento del Chocó.

\section{MATERIALES Y MÉTODOS}

Área de estudio. Las zonas estudiadas se localiza en las llanuras aluviales, colinas bajas y piedemonte cercanos al valle del Río Atrato, en municipios aledaños a los alrededores de Quibdó, capital del departamento del Chocó, donde se concentra la mayor pluviosidad del andén del Pacífico y donde las formaciones selváticas se encuentran entre las más ricas del mundo (Cuatrecasas 1958; Forero \& Gentry 1989). Los puntos de nuestros están incluidos en las coordenadas geográficas $5^{\circ} 00^{\prime}-6^{\circ} 45^{\prime} \mathrm{N}$ y $77^{\circ} 15^{\prime}-$ $76^{\circ} 30^{\prime} \mathrm{W}$. La temperatura de las zonas de estudio está dividido en dos tipos temperatura megatermal bajo (23.5 a $25.7^{\circ} \mathrm{C}$ ) y temperatura megatermal $\left(25.7\right.$ a $\left.27.9^{\circ} \mathrm{C}\right)$. El 
promedio de la temperatura media anual es $23.5^{\circ} \mathrm{C}$ (Poveda et al. 2004).

El brillo solar presenta una media mensual de $139.5 \mathrm{~h}$. El régimen de distribución de la precipitación es de tipo bimodal-tetraestacional con un período de mayor concentración entre abril y octubre y una época de menor concentración de lluvia desde noviembre hasta marzo con un promedio mensual de $395.5 \mathrm{~mm}$. El monto anual (promedio multianual) de la precipitación es $8558 \mathrm{~mm}$, con un valor extremo en Tutunendó con 11770 mm (Rangel \& Lowy 1993; Eslava 1994). Según Poveda et al. (2004) las zonas objeto de estudio están clasificadas dentro del régimen de Pluviosidad Alta y Muy Alta, ubicadas en el rango $8494-13670 \mathrm{~mm}$.

No se cuenta con un estudio general ni con aproximaciones sobre los tipos de vegetación en la zona. Las referencias a las comunidades vegetales se relacionan con las contribuciones de Cuatrecasas (1958) y del IGAC-Inderena-CONIF (1984).

En el valle del Río Atrato, se establecieron cinco municipios de la selva pluvial central, que presentan bosque pluvial tropical (bp-T); estas áreas de muestreos son: el municipio de Unión Panamericana "corregimiento de Salero", municipio de Certegui “Recta Larga”, municipio de Lloró “Centro Multipróposito de la Universidad Tecnológica del Chocó", municipio del Atrato "corregimiento de Samurindo” y municipio de Quibdó “área Suburbana en la cuenca hídrica del río Cabí”.

Se establecieron tres sitios de muestreos dentro de las áreas de estudios seleccionadas, caracterizados por presentar heterogeneidad en la cobertura vegetal, encontrándose bosques prístinos, bosque secundario y áreas de gestión antrópica (minería, agricultura, pastos etc.). Se realizaron 15 muestreos (tres en cada una de las cinco localidades) durante 15 meses, el estudio tuvo una duración de tres años (2008 a 2010), con una intensidad de ocho días por muestreo, estos se realizaron en horarios diurnos con un esfuerzo de muestreo de ocho horas/hombre, repartidas en las horas de la mañana, horas de la tarde y en ocasiones en las horas de la noche, donde se hicieron recorridos diarios aplicando el método de búsqueda libre sin restricción a lo largo de caminos abiertos al interior de cada uno de los sitios de muestreo.

Toma de información biológica. Los muestreos se realizaron por Encuentros Visuales (Visual Encounter Survey, VES), la cual es una técnica utilizada en el inventario y/o monitoreo de anfibios y reptiles que permite determinar la riqueza de especies en un área y estimar las abundancias relativas de las especies en un ensamblaje (Crump \& Scott 1994). Las capturas se realizaron con base en Rengifo (2002) y Paéz et al. (2002), buscando en las diferentes coberturas vegetales: árboles, arbustos y demás formaciones vegetales, y se inspeccionó en el interior de los diferentes sitios de muestreo, en lugares abiertos (caminos, suelos, bordes de quebradas, senderos y otros) y sitios de difícil acceso (bejuco, enredaderas, ramas altas de los árboles). En el momento de la captura a cada individuo se le tomo datos (hábitats, merísticos y morfométricos) y se mantuvieron en bolsas de tela hasta el procesamiento en el lugar de trabajo donde se hicieron registros fotográficos en vivo, para su posterior liberación.

Hábitats y microhábitats. Para la caracterización de la distribución espacial, se tuvieron en cuenta algunos datos ecológicos sugeridos por Heyer et al. (1994), como son la posición vertical (altura desde el suelo o agua) tomada de forma cualitativa así: suelo $(0 \mathrm{~cm})$, baja (<49 cm), media $(50-149 \mathrm{~cm})$, alta $(>150 \mathrm{~cm})$. También se registró el tipo de sustrato en el que se encontró el individuo en el momento de la captura, éste se dividió en cinco categorías: hojas, hojarascas, raíz, tronco y ramas. Además, se analizó estructuralmente el hábitat, combinando la posición vertical y el sustrato ocupado o usado por la misma en el instante del avistamiento.

Diámetro de percha. Se evaluó el diámetro de percha o diámetro del sitios exactos donde se registraron los individuos, usando cintas métricas que simularon una forcípula y se establecieron rangos desde $<5 \mathrm{~cm}$ hasta $>30 \mathrm{~cm}$; para el caso de las hojas los individuos fueron registrados en la categoría $<5 \mathrm{~cm}$.

Ecomorfos. Para determinar la relación del microhábitat usado por las especies de Anolis registradas en este estudio con su morfología (ecomorfo) se procedió a tomar datos morfométricos a los individuos según Velasco \& Herrel (2007) los cuales son: 1. Longitud hocico ano (LHA); 2. Longitud del húmero derecho (LHD; 3. Longitud Ulna (LU); 4. Longitud de la tibia derecha (LTD; 5. Longitud Fémur (LF); 6. Largo del cuarto dedo derecho (LDD), 7. Longitud Tronco (LTr); 8. Longitud Cola (LC); 9. Longitud Brazo (LB); 10 Longitud Pata (LP); 11 Longitud Ancho de la cabeza (LCAB); 12 Lamelas primera Falange (II) y Lamelas segunda Falange (III); todas estas medidas fueron tomadas con una calibrador digital con una precisión de $0.1 \mathrm{~mm}$, estas medidas fueron tomadas en dos ocasiones, si existía diferencia en las medidas eran reconfirmadas con una tercera, tal como lo recomiendan algunos autores (Irschick et al. 1997; Bickel \& Losos 2002).

Actividad, endemismos y estado de conservación. La actividad se determinó mediante el registro del comportamiento de los individuos en el momento de su captura o registro. La identificación de posibles especies endémicas y estado de conservación se realizó mediante la comparación del listado de especies registradas en este estudio, con la revisión de bibliografía de trabajos realizados en la 
región para el grupo faunístico (Castaño et al. 2004) listas de la Unión Internacional para la Conservación de la Naturaleza (UICN) y libros rojos de especies de reptiles.

Variables climáticas. En cada una de las áreas de muestreo se tomaron datos de temperatura y humedad relativa, por medio de un medidor de clima Kestrel 3000 y también los datos de la estación meteorología de la Universidad Tecnológica del Chocó (UTCH).

Determinación taxonómica. La determinación taxonómica se realizó en el Laboratorio de Zoología de la Universidad Tecnológica del Chocó con la ayuda de guías de campo y claves taxonómicas de Peters \& Donoso-Barros (1970), Rengifo \& Lundberg (1999) y Páez et al. (2002). Los ejemplares con dudas taxonómicas fueron identificados en el Laboratorio de Zoología de la Universidad del Valle.

Análisis de la información. Se hicieron pruebas de Chicuadrado para establecer la existencia de diferencias significativas en la distribución vertical, sustratos y diámetro de percha de las especies de Anolis dentro de los hábitats estudiados. Estas pruebas se realizaron con el programa SPSS Visauta (1998). Se diseñaron tablas bifactoriales entre la distancia vertical, sustratos, diámetro de percha con las abundancias de especies con Excel (2003) para Windows XP.

Se calculó la frecuencia como el número de apariciones por especie en cada área; la frecuencia relativa como el número de apariciones por especie sobre el total de apariciones por área, la densidad como el número total de la especie en cada área; la densidad relativa como el número total de la especie sobre el total de la densidad en cada área; el índice de valor de importancia (IVI) se calculó con la suma de la frecuencia relativa y la densidad relativa expresada en porcentaje (Curtis \& Intosh 1951). Para calcular el índice de jerarquización (IDJ) de la comunidad de Anolis presente en el corregimiento de Salero se tomó el valor más alto del IVI y se calculó el porcentaje de todos los demás valores a partir de él.

Se realizaron correlaciones de Pearson entre las variables climáticas de los hábitats con respecto a las abundancias de las especies de Anolis, usando el programa Excel para Microsoft Office (2003).

Análisis Multivariado. Se transformaron los datos morfológicos en $\log _{10}(\mathrm{x})$ para que cumplieran los supuestos de normalidad y homogeneidad; para probar diferencias entre las especies según las variables independientes (morfometría) de las especies reportadas en la selva pluvial central del Chocó, se realizó un análisis discriminante a 13 variables morfológicas; se realizó una ANOVA de una vía, con el propósito de probar diferencia significativa entre las especies según el sexo y una prueba de Tukey para determinar entre que grupos hubo diferencia.

\section{RESULTADOS}

Se registraron 303 individuos empleando un esfuerzo de muestreo de 960 horas/hombre, para un éxito de captura de 0.31 individuos/horas hombre. Este ensamble de Anolis se encuentra representado por diez especies, siendo la más representativas por su abundancia $A$. maculiventris con el 54.1\% ( $n=164)$, seguida de A. granuliceps con $20.1 \%(n=61)$ y A. chloris con $11.2 \%(n=34)$, estas especies en conjunto representan el $85.5 \%$ de la abundancia de Anolis registrada en este estudio (Cuadro 1).

Altura de percha. Se encontró una diferencia estadística $\left(X^{2}=58.7, \mathrm{gl}=3, P<0.0001\right)$ entre las cuatro categorías evaluadas, según la altura de percha o cuerpo de agua, donde el mayor registro de especies fue en la categoría

Cuadro 1. Posición vertical y ubicación por sustratos de las especies de Anolis en tres áreas con diferentes grados de intervención, Bosque Pluvial Tropical (bp-T), departamento del Chocó, Colombia.

\begin{tabular}{|c|c|c|c|c|c|c|c|c|c|}
\hline \multirow[t]{2}{*}{ Especie } & \multicolumn{4}{|c|}{ Posición Vertical } & \multicolumn{5}{|c|}{ Sustratos } \\
\hline & Suelo & Baja & Media & Alta & Hoja & Hojarasca & Raíz & Tronco & Rama \\
\hline Anolis maculiventris & 9 & 46 & 88 & 21 & 23 & 9 & 5 & 98 & 29 \\
\hline Anolis malkini & 0 & 1 & 1 & 2 & 0 & 0 & 0 & 2 & 2 \\
\hline Anolis chloris & 0 & 0 & 5 & 29 & 0 & 0 & 0 & 32 & 2 \\
\hline Anolis lyra & 0 & 1 & 3 & 1 & 0 & 0 & 0 & 4 & 1 \\
\hline Anolis biporcatus & 0 & 0 & 0 & 2 & 0 & 0 & 0 & 2 & 0 \\
\hline Anolis anchicayae & 0 & 4 & 6 & 4 & 2 & 0 & 0 & 8 & 4 \\
\hline Anolis chocorum & 0 & 0 & 7 & 4 & 0 & 0 & 0 & 11 & 0 \\
\hline Anolis notopholis & 0 & 0 & 0 & 4 & 0 & 0 & 0 & 3 & 1 \\
\hline
\end{tabular}


media con 39.6\% ( $n=120)$ de los individuos registrados, siendo A. maculiventris la mejor representada en esta posición con 73.3\% $(n=88)$ de los individuos, que también presentó diferencias estadísticamente significativas entre las categorías o posiciones para esta especie $\left(X^{2}=89.22\right.$, $\mathrm{gl}=3, P<0.0001)$. Las categorías bajo y alto $(n=80$ y 77$)$ siguieron en registros, y la categoría suelo solo reportó 29 individuos de las especies, siendo A. maculiventris y A. granuliceps exclusivas de esta categoría (Cuadro 1, Fig. 1). Sustrato de pecha. Se encontró una diferencia estadística entre los sustratos evaluados, según la abundancia de las especies $\left(\mathrm{X}^{2}=272.1, \mathrm{gl}=4, P<0.0001\right)$, lo cual se debe a que en el sustrato tronco registró la mayor abundancia de individuos con el 57.4\% $(n=174)$ siendo A. maculiventris la mejor representada en esta posición con 56.3\% ( $n=$ 98) de los individuos, presentando diferencias estadísticamente significativa entre los sustratos evaluados para esta especie $\left(\mathrm{X}^{2}=89.22, \mathrm{gl}=3, \mathrm{P}<0.0001\right.$ ) ( Cuadro 1 ).

En cuanto a la exclusividad de sustrato, A. biporcatus y A. chocorum concurrieron únicamente en tronco, la primera fue una especie rara en este estudio porque sólo se registraron dos individuos que imposibilitan el conocimiento de sus preferencias de hábitat y en el caso de A. chocorum es una especie que por su tamaño requiere sustratos consistentes que permita el soporte de su peso corporal. Otras especies no exclusivas pero que presentaron una alta ocurrencia en el sustrato tronco fueron $A$. notopholis y A. chloris con 75 y $94.1 \%$ de los individuos respectivamente.

Análisis estructural del hábitat. Se realizó el análisis estructural del hábitat ocupado por las especies de Ano- lis y se observó que este grupo de organismos presentó mayor frecuencia de ocurrencia en los estratos medios y bajos altos de la cobertura vegetal ( $n=120$ y 80 individuos, respectivamente), lo cuales se ubican en el sustrato tronco y rama de dichas coberturas vegetales $(n=174 \mathrm{y}$ 50 individuos, respectivamente), seguido de los estratos altos y los sustratos hojarascas, hoja y raíz con muy pocas especies. El suelo fue la posición con menor registro de individuos, en donde sólo se registraron $A$. maculiventris y A. granuliceps (Cuadro 2, Fig. 2).

Diámetro de percha. Las categorías evaluadas presentaron diferencia significativa $\left(\mathrm{X}^{2}=147.5, \mathrm{gl}=6, P<00001\right)$ ya que los rangos $<5(n=82), 6-10(n=83)$ y $11-15(n$ = 67) tuvieron las mayores abundancias, representados por Anolis maculiventris, A. granuliceps, A. notopholis, A. chloris y A. anchicayae. Todas estas especies son del grupo de Anolis pequeños que pueden perchar en árboles y arbustos de diámetros muy pequeños (Cuadro 3).

Índice de valor de importancia (IVI). Anolis maculiventris y A. anchicayae fueron las especies que presentaron mayor IVI con 0.69 y 0.51 , a nivel proporcional 33 y $25 \%$, respectivamente. En el área de bosque secundario, las especies $A$. maculiventris y A granuliceps fueron las de mayor IVI con 0.80 y 0.66 , con porcentaje de 40 y $33 \%$ de la comunidad reportada en esta zona, y en las zonas de gestión, A. maculiventris con 0.95 fue la más representativa (Cuadro 4).

Índice de jerarquización (IDJ). En bosque, el mayor IDJ fue para $A$. maculiventris con $100 \%$, seguido de $A$. anchicayae con $75 \%$, en la zona de bosque secundario la especie con mayor IDJ fue A. granuliceps con 100\%,



Figura 1. Media y desviación estándar de la posición vertical de las especies de Anolis en tres áreas con diferentes grados de intervención, Selva Pluvial Tropical, departamento del Chocó, Colombia. 




Figura 2. Distribución estructural del microhábitat ocupado por las especies de Anolis en tres áreas con diferentes grados de intervención, Selva Pluvial Tropical, departamento del Chocó, Colombia.

Cuadro 2. Distribución estructural del microhábitat ocupado por las especies de Anolis en tres áreas con diferentes grados de intervención, Selva Pluvial Tropical, departamento del Chocó, Colombia.

\begin{tabular}{|c|c|c|c|c|c|c|}
\hline \multirow[t]{2}{*}{ Sustrato } & \multicolumn{4}{|c|}{ Distribución Vertical (altura) } & \multirow[t]{2}{*}{ Total } & \multirow[t]{2}{*}{$(\%)$} \\
\hline & Suelo $(0 \mathrm{~cm})$ & Bajo $(<49 \mathrm{~cm})$ & Medio $(50-149 \mathrm{~cm})$ & Alto $(>150 \mathrm{~cm})$ & & \\
\hline Hoja & 0 & 6 & 13 & 7 & 26 & 8.6 \\
\hline Hojarasca & 25 & 0 & 1 & 0 & 26 & 8.6 \\
\hline Raíz & 1 & 17 & 9 & 0 & 27 & 8.9 \\
\hline Tronco & 0 & 37 & 74 & 63 & 174 & 57.4 \\
\hline Rama & 0 & 20 & 23 & 7 & 50 & 16.5 \\
\hline Abundancia & 26 & 80 & 120 & 77 & 303 & 100 \\
\hline
\end{tabular}

seguido de $A$. chocorum con $84 \%$, y en el área de gestión correspondió a A. maculiventris con $100 \%$ y A. granuliceps con $49 \%$ como la segunda especie de mayor jerarquía en dicha área.

Ecomorfo del ensamble de Anolis. En este estudio sobresalieron las especies de ecomorfo tronco-rama Anolis malkini, A. chloris, A. latifrons, A. lyra y A. notopholis; todas las especies ocurren en los troncos de los árboles y arbustos. Especies como A. maculiventris y A. granuliceps se pueden encontrar ocupando microhábitat en el suelo generalmente en los troncos caídos y la hojarasca (Cuadro 5).

El cuadro 6 muestra las medias y la desviación estándar de 13 variables morfométricas de las 10 especies de Anolis reportadas en zonas de bosque pluvial tropical en la zona centro del departamento del Chocó. Según análisis de varianza realizado a la variable LRC, las especies fueron significativamente diferentes (Wilk's, $\mathrm{F}_{7,105}$ = 77.97, $P<0.0001$ ), siendo $A$. latifrons la que expone mayor variación morfológica en seis de las siete funcio- nes discriminantes que presentaron valores significativos a un nivel de confianza del 95\%. Las funciones LRC y LTR explican el 79.1\% de la varianza expresada en las ocho especies (Cuadro 7, Fig. 3), y se observa como A. latifrons se separa del resto de las especies.

El análisis de correlación entre variables independientes, muestra como las medidas corporales LTR y LRC (0.82) se correlacionan altamente en todas las especies, seguido de las variables, LRC vs ANCAB (0.75), LTR vs ANCAB (0.71), LB vs LP (0.71), H vs T (0.72), F vs T (0.76), F vs ANCAB (0.71), ANCAB vs ALCAB (0.71).

Variables climáticas. La temperatura presentó valores mínimos de $23.3{ }^{\circ} \mathrm{C}$ en la zona de bosque que tiene de 30 a 60 años de regeneración natural. Una temperatura máxima de $27.6^{\circ} \mathrm{C}$ fue registrada en áreas de gestión. La humedad relativa presentó valores mínimos de $83.2 \%$ en el área de gestión y valores máximos $98.4 \%$ en bosque en regeneración actual (Fig. 4 y 5).

El análisis de la abundancia de Anolis con las variables climáticas temperatura y humedad relativa muestra 
Cuadro 3. Diámetro de Percha de un ensamble de Anolis en tres áreas con diferentes grados de intervención, Selva Pluvial Tropical, departamento del Chocó, Colombia.

\begin{tabular}{|c|c|c|c|c|c|c|c|c|}
\hline \multirow[t]{2}{*}{ Especies } & \multicolumn{7}{|c|}{ Diámetro de Percha (cm) } & \multirow[t]{2}{*}{ Total } \\
\hline & $>5$ & $6-10$ & $11-15$ & $16-20$ & $21-25$ & $26-30$ & $<30$ & \\
\hline Anolis maculiventris & 59 & 48 & 35 & 22 & 0 & 0 & 0 & 164 \\
\hline Anolis granuliceps & 23 & 27 & 11 & 0 & 0 & 0 & 0 & 61 \\
\hline Anolis malkini & 0 & 0 & 0 & 0 & 1 & 1 & 2 & 4 \\
\hline Anolis chloris & 0 & 5 & 8 & 0 & 6 & 3 & 12 & 34 \\
\hline Anolis latifroms & 0 & 0 & 0 & 0 & 0 & 0 & 4 & 4 \\
\hline Anolis lyra & 0 & 0 & 0 & 0 & 1 & 4 & 0 & 5 \\
\hline Anolis biporcatus & 0 & 0 & 1 & 1 & 0 & 0 & 0 & 2 \\
\hline Anolis anchicayae & 0 & 2 & 8 & 3 & 1 & 0 & 0 & 14 \\
\hline Anolis chocorum & 0 & 0 & 1 & 1 & 6 & 3 & 0 & 11 \\
\hline Anolis notopholis & 0 & 1 & 3 & 0 & 0 & 0 & 0 & 4 \\
\hline
\end{tabular}

Cuadro 4. Valor de importancia ecológica de la comunidad de Anolis en tres áreas con diferentes grados de intervención, Selva Pluvial Tropical, departamento del Chocó, Colombia. IVI: índice de valor de importancia, IVIP: índice de valor de importancia relativo.

\begin{tabular}{|c|c|c|c|c|c|c|}
\hline \multirow[t]{2}{*}{ Especies } & \multicolumn{2}{|c|}{ Zona I } & \multicolumn{2}{|c|}{ Zona II } & \multicolumn{2}{|c|}{ Zona III } \\
\hline & IVI & IVIP & IVI & IVIP & IVI & IVIP \\
\hline A. chloris & 0.046 & 0.022 & 0.122 & 0.061 & 0.44 & 0.22 \\
\hline A. granuliceps & 0.092 & 0.044 & 0.669 & 0.335 & 0.468 & 0.234 \\
\hline A. latifrons & 0.046 & 0.054 & 0.122 & 0.061 & 0 & 0 \\
\hline A. chocorum & 0.248 & 0.12 & 0.204 & 0.102 & 0 & 0 \\
\hline A. malkini & 0.046 & 0.022 & 0.041 & 0.02 & 0.056 & 0.028 \\
\hline A. anchicayae & 0.516 & 0.25 & 0.041 & 0.02 & 0 & 0 \\
\hline A. notopholis & 0.157 & 0.076 & 0 & 0 & 0 & 0 \\
\hline
\end{tabular}

Cuadro 5. Categorías de uso de microhabitat, altura de percha y ecomorfo de la comunidad de Anolis en tres coberturas vegetales en la selva pluvial tropical en la zona centro del Chocó, Colombia.

\begin{tabular}{llcl}
\hline \multicolumn{1}{c}{ Especie } & \multicolumn{1}{c}{ Microhábitat } & Altura de Percha (m) & Ecomorfo \\
\hline Anolis maculiventris & Arbóreo (Tronco-rama) y Suelo (Raíz-hojarasca) & $0-3$ & Tronco-arbusto Raíz-hojarasca \\
Anolis granuliceps & Suelo (Raíz-hojarasca) Arbóreo (Tronco-rama) & $0-3$ & Tronco-arbusto Raíz-hojarasca \\
Anolis malkini & Arbóreo (Tronco-rama) & $0.50-5$ & Tronco-rama \\
Anolis chloris & Arbóreo (Tronco-rama) & $0.50-5$ & Tronco-rama \\
Anolis latifrons & Arbóreo (Tronco-rama) & $1-5$ & Tronco-rama \\
Anolis lyra & Arbóreo (Tronco-rama) & $0-5$ & Tronco-rama \\
Anolis biporcatus & Arbóreo (Tronco) & $1-5$ & Tronco \\
Anolis anchicayae & Arbóreo (Tronco-rama, hojas) & $0-5$ & Tronco-rama-arbusto \\
Anolis chocorum & Arbóreo (Tronco) & $0-5$ & Tronco \\
Anolis notopholis & Arbóreo (Tronco-rama) & $0-3$ & Tronco-rama \\
\hline
\end{tabular}

una relación lineal negativa débil para el bosque $\left(R^{2}=-\right.$ 0.05 ), pero positiva en el bosque en regeneración actual $\left(R^{2}=0.0032\right)$ y en el área de gestión $\left(R^{2}=0.0859\right)$ (Figs. 6 y 7$)$.

\section{DISCUSIÓN}

El ensamble del género Anolis en zonas de bosque pluvial y muy húmedo tropical en el departamento del Chocó, 





Cuadro 7. Matriz de Factores y Funciones discriminantes de especies de Anolis de la zona centro del departamento del Chocó, Colombia. Ver en el texto el significado de las abreviaturas.

\begin{tabular}{|c|c|c|c|c|c|c|c|}
\hline \multirow[t]{2}{*}{ Factores } & \multicolumn{7}{|c|}{ Funciones discriminantes } \\
\hline & 1 & 2 & 3 & 4 & 5 & 6 & 7 \\
\hline LRC & -0.24225 & -0.48244 & -0.08896 & 0.05917 & 0.05738 & 0.93874 & -0.81322 \\
\hline $\mathrm{LTr}$ & 0.05899 & -0.51573 & -0.84965 & -0.47269 & -0.07038 & -0.30610 & 0.18489 \\
\hline LC & 0.03877 & 0.02395 & -0.32552 & -0.29028 & 0.14824 & 0.13293 & 0.61803 \\
\hline LB & 0.14985 & -0.61374 & 0.11144 & 0.32431 & -0.57224 & -0.18221 & 0.16797 \\
\hline LP & -0.30034 & 0.44379 & 0.32670 & 0.13005 & -0.42931 & 0.47337 & -0.16628 \\
\hline $\mathrm{H}$ & 0.00871 & -0.34518 & 0.19240 & -0.75576 & -0.43440 & -0.47701 & -0.18682 \\
\hline $\mathrm{U}$ & 0.29945 & -0.08371 & -0.00038 & 0.62606 & 0.24397 & -0.19628 & 0.92458 \\
\hline $\mathrm{F}$ & 0.34630 & 0.78004 & -0.40867 & -0.75359 & -0.45502 & -0.09390 & 0.29255 \\
\hline $\mathrm{T}$ & -0.11278 & 0.56404 & -0.55250 & 0.92724 & -0.19214 & 0.71689 & -0.48618 \\
\hline D4 & 0.45010 & 0.37655 & -0.16836 & 0.24135 & 0.02049 & -1.20988 & -0.17711 \\
\hline ANCAB & 0.70375 & -0.30587 & 1.35282 & 0.29618 & 0.49818 & -0.72901 & -0.01065 \\
\hline ALCAB & 0.32087 & 0.61682 & -0.07032 & -0.50100 & 0.79605 & 0.34534 & 0.03426 \\
\hline LCAB & -0.56611 & -0.59806 & 0.04192 & 0.22470 & 0.05714 & 0.54737 & -0.11060 \\
\hline
\end{tabular}



Figura 3. Análisis de funciones discriminantes de especies de Anolis de la zona centro del departamento del Chocó, Colombia.

albergan una significativa riqueza de este género, siendo la cobertura vegetal bosque, la que ofrece mejores condiciones para las especies (Rengifo et al. 2014). La heterogeneidad de la vegetación que presentan estos bosques en la región natural chocoana juega un papel importante en la diversidad, ya que proporciona una mayor variedad de microhábitats para este ensamble de estos reptiles, considerados como los más diversos y adaptables que constituyen la fauna de vertebrados terrestres que habitan en los bosques tropicales (Pleguezuelos \& Fetiche 2004).

Dentro de esta heterogeneidad, los Anolis presentaron mayor frecuencia de ocurrencia en los estratos medios de las coberturas vegetales, sin importar el tipo de ambientes, concordando con Páez et al. (2002) en el sentido de que los Anolis son un grupo que se puede adaptar a una gran diversidad de biotopos y con Duellman (1979), quien sostiene que los reptiles en las selvas tropicales exploran los hábitats arbóreo, terrestre, acuático o semiacuático, utilizando todos los recursos en términos de vegetación. El proceso de selección de hábitat por parte de los individuos de Anolis, está regido por elegir determinados recursos espaciales disponibles en el ambiente (Partridge 1978; Garshelis 2000), y depende de la estructura física del ambiente, la fisiología del animal, la disponibilidad de alimento y la protección contra depredadores (Reaney \& Whiting 2003).

La coloración críptica de algunas de especies de Anolis hace posible la exploración de los estratos bajos y medios 


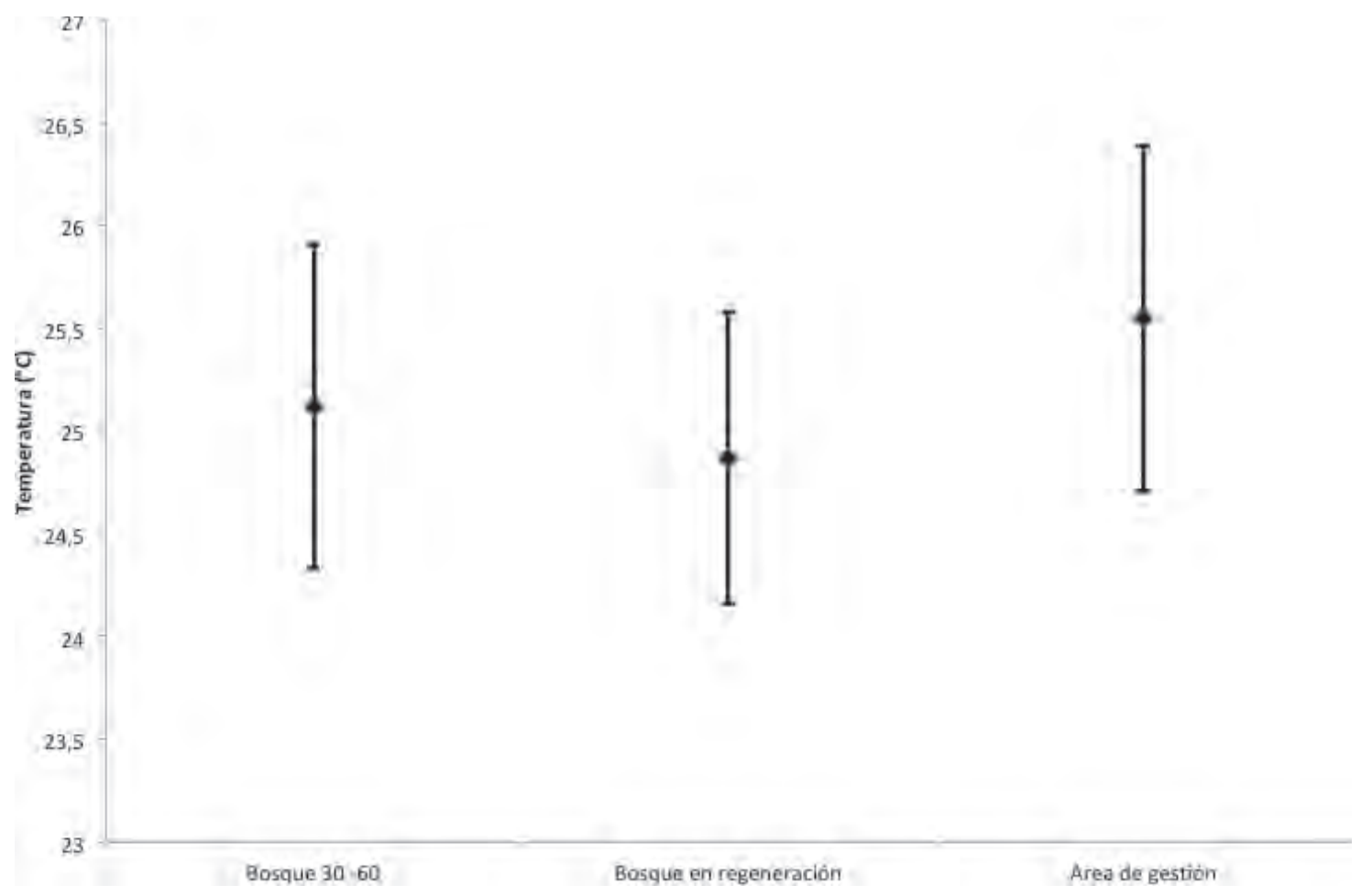

Figura 4. Promedio y desviación estándar de los valores de temperatura, registrados en tres áreas con diferentes grados de intervención, Selva Pluvial Tropical, departamento del Chocó, Colombia.



Figura 5. Promedio y desviación estándar de los valores de humedad, registrados en tres áreas con diferentes grados de intervención, Selva Pluvial Tropical, departamento del Chocó, Colombia.

de los hábitats o microhábitats disponibles en los bosques tropicales. Sin embargo, se registraron un gran número de individuos de A. chloris, A. notopholis, A. biporcatus y $A$. chocorum en los estratos altos de las estructuras vegetales. Este hábito facilita el escape de sus depredadores, les permite asechar a sus presas y percharse para descansar.

En términos de sustrato de percha los Anolis por sus características estructurales prefieren principalmente troncos desnudos de los árboles, es decir sin la presen- 



Figura 6. Regresión lineal entre la temperatura y la abundancia de Anolis registrados en tres áreas con diferentes grados de intervención, Selva Pluvial Tropical, departamento del Chocó, Colombia.

cia de epifitas, musgos, bejucos o lianas. A. maculiventris fue la especie que se encontró en todos los sustratos evaluados y A. granuliceps solo no se registró en el sustrato hoja, probablemente debido a la coloración opaca que presentan estos animales, en donde puede ser más conspicuos para los depredadores. En cambio, A. maculiventris presenta una coloración críptica, pero puede camuflar perfectamente en diferentes sustratos (Velasco \& Herrel 2007).
Los Anolis son de actividad diurna, en este periodo del día los individuos se observaron alimentándose y en locomoción, y en el caso de A. maculiventris, A. granuliceps y A. chloris algunas veces en cortejo. Estas tres especies son relativamente fáciles de observar ya que exploran rangos bajos de altura y en una variedad de sustratos. A pesar que la gran mayoría de las especies de Anolis son muy territoriales solo se registró agresión en dos especies, A. lyra y A. chloris. En esta actividad, estas especies exhiben el 



Figura 7. Regresión lineal entre la humedad relativa y la abundancia de Anolis registrados en tres áreas con diferentes grados de intervención, Selva Pluvial Tropical, departamento del Chocó, Colombia.

abanico gular y realizan movimientos repetidos en forma de flexión de las extremidades anteriores; sin embargo, no se observaron ataques.

El registro de animales perchados está muy relacionada con la experiencia y capacidad de observación, y que tan conspicuos sean; por ejemplo A. malkini, A. latifrons, A. lyra y A. biporcatus fueron especies de poca frecuencia de ocurrencia en los muestreos, lo cual imposibilitó realizar un análisis sobre la altura y sitios percha, ya que esta puede ser una variable que depende de las condiciones ambientales, comportamiento de los animales y estado de excitación de los mismo, por lo que pocas observaciones no son concluyentes. En cambio A. chloris fue una de las especies más representativas en términos de abundancia en este estudio, $\mathrm{y}$ fue registrada en alturas superiores a los cinco metros y ocupando todos los estratos y una variedad de sustratos en una estructura arbórea.

Estructuralmente (altura y sitio de percha) el uso del hábitat de los Anolis está muy relacionado con la estructura arbórea. Estos reptiles tienen la capacidad de seleccionar hábitat con algunas características especiales, en los que algunas especies de árboles pueden ser considera- 
das como indicadoras de calidad del hábitat que ocupan, ya que requieren estructuras arbóreas que se recambian según las condiciones de los distintos ambientes. Diversos trabajos sobre uso de hábitat, han demostrado que la distribución espacial parece ser un fenómeno recurrente en especies simpátricas de lagartijas (Jenssen et al.1984; Reinert 1984; Rummel \& Roughgarden 1985; Goto \& Osborne 1989), este estudio ha demostrado como un ensamble de Anolis puede usar y distribuirse en coberturas vegetales en zonas de bosque pluvial y muy húmedo tropical en la zona centro del departamento del Chocó.

La diferencia encontrada al evaluar el diámetro de percha o sitios exactos donde se registraron los individuos, se relaciona con los ejemplares que usan las hojas como sitio de percha, siendo este incluido en categorías inferiores. Es importante anotar que las especies de Anolis prefieren diámetros de percha pequeños, en ramas desprovistas de epífitas, lianas y bejucos. Autores como Jenssen (1973), Kiester et al. (1975), Jenssen et al. (1998) y Vitt et al. (2002, 2003) han evaluado la selección y el uso de hábitat en lagartijas del género Anolis, demostrando que la altura, el diámetro y el microclima de la percha son características importantes en la distribución espacial-temporal entre las especies.

El índice de valor de importancia combina dos elementos que determinan el peso de una especie en cuanto el uso del hábitat del ensamble de Anolis en zonas de bosque pluvial y muy húmedo tropical. Este estudio mostró que $A$. maculiventris es la especie que mejor aprovecha los recursos en términos de hábitat en este ecosistema, ya que explora los recursos sin importar la cobertura vegetal, es decir, desde áreas intervenidas hasta los bosques maduros, además de ocupar las posiciones verticales (alturas) y los sustratos (sitio percha) disponibles. Esta especie es la más plástica en términos de uso del hábitat, lo que la hace tener una jerarquía en términos de abundancia y frecuencia con respecto al resto del ensamblaje. En comparación, A. anchicayae está restringida a los bosques maduros y ocasionalmente a bosque secundario en regeneración, y A. granuliceps tiene el mayor valor de importancia en bosque secundario, pero no es exclusiva de esta cobertura en la región centro del departamento del Chocó.

El esfuerzo de muestreo aplicado en esta investigación nos permite comentar sobre la ecología y principalmente sobre la forma de uso del hábitat (ecomorfo) y microhábitat (altura y sitio de percha) de las especies de Anolis registradas en las tres coberturas vegetales en la zona centro del departamento del Chocó. La misma estructura corporal de estas especies, favorecida por la presencia de lamelas para adherirse a los sustratos y escalar estructuras arbóreas, hace que estos animales se relacionen con el componente vegetal, prefiriendo árboles con las caracte- rísticas antes mencionadas, y usan desde las hojas y troncos lisos hasta suelo y raíces en descomposición. Otros estudios han confirmado que muchas especies del género Anolis exhiben una fuerte asociación entre su morfología y el tipo de microhábitat que utilizan (Pounds 1988; Losos 1990a, 1990b; Beuttell \& Losos, 1999), la cual se ve reflejada en diferencias en el uso de microhábitat por especies simpátricas cuya morfología y/o tamaño son diferentes (Pounds 1988; Losos \& de Queiroz 1997; Losos 1990b) y también en diferencias ontogénicas en el uso de hábitat dentro de una especie (Pounds 1988; Jenssen et al.1998; Irschick et al.2000).

El análisis discriminante muestra claramente como A. latifrons se separa del resto de las especies según las variables tenidas en cuenta, vislumbrando así variación morfológica. Esta especie de Anolis es de gran porte, como el resto de las especies que componen este grupo (Velasco 2007), que presentan una amplia distribución en el continente y en regiones insulares.

El análisis de la abundancia de Anolis con las variables climáticas medidas mostró una relación lineal negativa con el área de bosque con 30 a 60 años de regeneración y correlación débil con el bosque en regeneración actual y el área de gestión, debido a que las variables no presentaron mayores fluctuaciones durante los muestreos, con promedios no muy distanciados. Esto indica que estos hábitats o coberturas vegetales no presentan limitante en cuanto la temperatura y que la distribución de los Anolis no está influenciado por esta variable climática, excepto en el caso del área de gestión presenta muy poca cobertura vegetal y los árboles frutales presentes no brindan la suficiente cobertura vegetal y tiende a incrementarse la temperatura; además, A. maculiventris, A. granuliceps y A. chloris fueron mucho más abundantes en esta área y sus mayores abundancias están asociadas con la media de temperatura para esta área.

Un comportamiento muy similar ocurrió con la variable humedad relativa, donde el área de bosque con 30 a 60 años de regeneración no mostró una correlación muy fuerte, registrando la mayor abundancia en los valores medios para esta zona (92\%). El bosque en regeneración actual y el área de gestión mostraron una correlación negativa, con disminución en las abundancias a medida que se incrementaron los valores de la variable.

\section{LITERATURA CITADA}

Calsbeek, R. \& Irschick, D. J. 2007. The quick and the dead: correlational selection on morphology, performance, and habitat use in island lizards. Evolution, 61: 2493-2503.

Castaño, O., Cárdenas, G., Hernández, E. J. \& Castro, F. 2004. Reptiles en el Chocó Biogeográfico, pp. 277-324. In: J. O. Rangel. (Ed). Colombia Diversidad Biótica IV: El Chocó bio- 
geográfico/CostaPacífica.UniversidadNacionaldeColombia,Bogotá D.C.

Castro, F. 1988. Niche structure on an anole community in a tropical rain forest within the Chocó Region of Colombia. PhD Thesis, North Texas State University.

Crump, M. \& Scott, N. 1994. Visual encounter surveys, pp. 84-92. In: R. Heyer, M. Donnelly, R. McDiarmid, L. Hayek \& M. Foster (Eds.). Measuring and Monitoring Biological Diversity: standard methods for amphibians. Smithsonian Institution Press, Washington.

Cuatrecasas, J. 1958. Aspectos de la vegetación natural de Colombia. Revista de la Academia Colombiana de Ciencias, 10: 221-268.

Daza, R. J. 1997. Mastofauna y Herpetofauna Encontradas el Área de Influencia Directa del Proyecto Hidroeléctrico, Nechi-Antioquia.

Duellman, W. E. 1979. The South American Herpetofauna: Its Origin, Evolution and Dispersal. Monograph of the Museum of Natural History, University of Kansas, 7: 1-485.

Elstrott, J. \& Irschick, D. J. 2004. Evolutionary correlations among morphology, habitat use and clinging performance in Caribbean Anolis Lizards. Biological Journal of the Linnean Society, 83: 389-398.

Eslava, J. A. 1994. Climatología del Pacífico colombiano. Academia Colombiana de Ciencias Geofísicas. Coleccióon Eratóstenes, No. 1, Santa Fe de Bogotá D.C., 79 pp.

Estrada, A. R. \& Silva, A. 1984. Análisis de la ecomorfología de 23 especies de lagartos cubanos del género Anolis. Ciencias Biológicas, 12: 91-104.

Estrada, L. J. 1997. Estudio Preliminar de la Herpetofauna del Alto de San Miguel (Caldas- Antioquia).

Forero, E. \& Gentry, A. H. 1989. Lista anotada de las plantas del departamento del Chocó, Colombia. Biblioteca J.J. Triana 10 Instituto de Ciencias Naturales, Universidad Nacional de Colombia, $138 \mathrm{pp}$.

García, U. \& Mosquera, F. 2005. Caracterización Taxonómica de la Comunidad de Lagartos (Squamata-Lacertilia) en el Sotobosque de la Cuenca del Río Cabi, Choco. Tesis de grado. Choco - Colombia, Universidad Tecnológica del Choco “Diego Luís Córdoba”, Facultad de Ciencias. 60 pp.

Garshelis, D. L. 2000. Delusions in habitat evaluation: Measuring use, election, and importance, pp. 111-164. In: L. Boitani \& T. K. Fuller (Eds.). Research techniques in animal ecology, controversies and consequences. Columbia University Press, New York.

Halffter, G. 1994. Conservación de la biodiversidad: Un reto del fin de siglo. Boletín del Instituto Catalán de Historia Natural, 62: 137-146.

Heyer, W., Donnelly, M., McDiarmid, R., Hayek, L. \& Foster, M. 1994. Measuring and Monitoring Biological Diversity. Standard Methods for Amphibians. Smithsonian Institution Press, Washington, D.C. 364 pp.

IGAC, INDERENA, CONIF. 1984. Bosques de Colombia, memoria explicativa. Instituto Geográfico “Agustín Codazzi”. Teledetección.

Jackman, T., Losos, J. B., Larson, A. \& De Queiroz, K. 1997. Phylogenetic studies of convergent adaptive radiations in Caribbean Anolis lizards, pp. 535-557. In: T. J. Givnish and K. J. Sytsma (Eds.). Molecular evolution and adaptive radiation. Cambridge University Press, Cambridge.

Jenssen, T. A. 1973. Shift in the structural habitat of Anolis opalinus due to congeneric competition. Ecology, 54:863-869.

Jenssen, T. A., Hovde, K. A. \& Taney, K. G. 1998. Size-related habitat use by nonbreeding Anolis carolinensis lizards. Copeia, 3:774-779.
Kiester, A. R., Gorman, G. C. \& Arroyo, D. C. 1975. Habitat selection behavior of three species of Anolis lizards. Ecology, 56:220-225.

Leal, M., Rodriguez, J. A. \& Losos, J. B. 1998. An Experimental study of Interspecific Interaction between two Puerto Rican Anolis Lizards. Oecologia, 117: 273-278.

Losos, J. B, Jackman, T. R., Larson, A., De Queiroz, K. \& Rodríguez-Schettino, L. 1998. Historical Contingency and determinism in replicated adaptive radiations of island lizards. Science, 279: 2115-2118.

Losos, J. B. \& Sinervo, B. 1989.The effects of morphology and perch diameter on sprint performance of Anolis lizards. Journal of Experimental Biology, 245: 23-30.

Moermond, T. C. 1979. Habitat constraints on the behavior, morphology, and community structure of Anolis lizards. Ecology, 60: 152-164.

Molina, C. \& Gutiérrez- P. D. A. 2007. Uso nocturno de perchas en dos especies de Anolis (Squamata:Polychrotidae) en un bosque Andino de Colombia. Papéis Avulsos de Zoologia, 47: 273-281.

Páez, V. P., Brian, C. B., Estrada, J. J., Ortega, A. M., Daza, J. M. \& Gutiérrez-C., P. D. 2002. Guía de campo de algunas especies de anfibios y reptiles de Antioquia. Universidad Nacional de Colombia, Conciencias, Universidad de Antioquia, Medellín. $137 \mathrm{pp}$.

Partridge, L. 1978. Habitat selection, pp. 351-376. In: J. R. Krebs \& N. B. Davies (Eds.). Behavioural ecology: an evolutionary approach. Sinauer Associates, Sunderland, Massachusetts.

Peters, J. A. \& Donoso, B. R. 1970. Catalogue of the Neotrpical Squamata part I. Lizards and Amphisbaenia. Bulletin of the United States National Museum, 297: 1-293.

Pounds, J. A. 1988. Ecomorphology, locomotion, and microhabitat structure: patterns in a tropical mainland Anolis community. Ecological Monographs, 58: 299-320.

Poveda, M. C., Rojas, C. A., Ruedas, A. \& Rangel, J. O. 2004. El Chocó biogeográfico: Ambiente físico. pp. 1-21. In: J. O. Rangel. (Ed). Colombia Diversidad Biótica IV: El Chocó biogeográfico/ Costa Pacífica. Universidad Nacional de Colombia, Bogotá D.C.

Rangel-Ch. J.O. \& Lowy-C., P.D. 1993. Tipos de vegetación y rasgos fitogeográficos, pp. 184-198. In: P. Leyva, (Ed). Colombia Pacifico Tomo I. Editorial del Fondo FEN. Instituto de Ciencias Naturales, Universidad Nacional de Colombia. Bogotá.

Reaney, L. T. \& Whiting, M. J. 2003. Picking a tree: habitat use by the tree agama, Acanthocercus atricollis atricollis, in South Africa. African Zoology, 32:273-278.

Rengifo, J. T. 2002. Composición y estructura de la comunidad de reptiles presente en dos zonas del bosque pluvial tropical en el departamento del Chocó. Trabajo de grado como requisito para optar al título de Biólogo con Énfasis en Recursos Naturales. Universidad Tecnológica del Choco Diego Luis Córdoba, Quibdó. 65 pp.

Rengifo, J. T., Castro, F. \& Purroy, F. J. 2014. Diversidad de una comunidad de Anolis en la selva pluvial central en el departamento del Chocó, Colombia. Basic \& Applied Herpetology (online frist: https://sites.google.com/site/bahonlinesite/online-first/12004).

Renjifo, J. M. \& Ludberg, G. 1999. Guía de Campo; Anfibios y Reptiles de Urrá. Editorial Colina. Medellín, Colombia.

Traverzo-Pérez, F. 2008. Hábitat estructural nocturno y fidelidad al dormidero en Anolis cristatellus y Anolis krugi. Tesis sometida en cumplimiento parcial de los requisitos para el grado de Maestro en Ciencias en Biología, Universidad de Puerto Rico, Recinto Universitario de Mayagüez. 
Vargas, S \& Bolaños, M. 1999. Anfibios y Reptiles Presentes en Hábitats Perturbados de la Selva Lluviosa Tropical en el Bajo Anchicaya, Pacifico Colombiano. Revista de la Academia Colombiana de Ciencias, 23(suplemento especial): 499-511.

Velasco, J. A. \& Herrel, A. 2007. Ecomorphology of Anolis Lizards of the Chocó region in Colombia and comparisons with greater Antillaean ecomorphs. Biological Journal of the Linnean Society, 92: 29-39.

Vitt, L. J., Ávila-Pires, T. C., Espósito, M. C., Sartorius, S. S. \& Zani, P. A. 2003. Sharing Amazonian rain-forest trees: Ecology of Anolis punctatus and Anolis transversalis (Squamata: Polychrotidae). Journal of Herpetology, 37:276-285.
Vitt, L. J., Ávila-Pires, T. C., Zani, P. A. \& Espósito, M. C. 2002. Life in shade: The ecology of Anolis trachyderma (Squamata:Polychrotidae) in Amazonian Ecuador and Brazil, with comparisons to ecologically similar Anoles. Copeia, 2:275-286.

Williams, E. E. 1976. West Indian anoles: A taxonomic and evolutionary summary. I. Introduction and a species list. Breviora, 440: $1-21$.

Williams, E. E. 1983. Ecomorphs, faunas, island size, and diverse end points in island radiations of Anolis, pp. 326-370. In: R. B. Huey, E. R. Pianka \& T. W. Schoener (Eds.). Lizard ecology: Studies of a model organism. Harvard University Press, Cambridge. 